\title{
Exploiting knowledge composition to improve real-life hand prosthetic control
}

\author{
Gauravkumar Patel*, Markus Nowak and Claudio Castellini Member,IEEE
}

\begin{abstract}
In myoelectric prosthesis control, one of the hottest topics nowadays is enforcing simultaneous and proportional $(\mathrm{s} / \mathrm{p})$ control over several degrees of freedom. This problem is particularly hard and the scientific community has so far failed to provide a stable and reliable s/p control, effective in daily-life activities. In order to improve the reliability of this form of control, in this work we propose on-the-fly knowledge composition, thereby reducing the burden of matching several patterns at the same time, and simplifying the task of the system. In particular, we show that using our method it is possible to dynamically compose a model by juxtaposing subsets of previously gathered (sample,target) pairs in real-time, rather than composing a single model in the beginning and then hoping it can reliably distinguish all patterns. Fourteen intact subjects participated in an experiment, where repetitive daily-life tasks (e.g. ironing a cloth) were performed using a commercially available dexterous prosthetic hand mounted on a splint and wirelessly controlled using a machine learning method. During the experiment, the subjects performed these tasks using myocontrol with and without knowledge composition and the results demonstrate that employing knowledge composition allowed better performance, i.e. reducing the overall task completion time by $30 \%$.
\end{abstract}

Index Terms-human-machine interfaces, hand prosthetics, machine learning, knowledge composition

\section{INTRODUCTION}

Reliability of myoelectric control is one of the most demanding challenges nowadays in assistive robotics, particularly when it comes to dexterous control of multifinger hand prostheses. The world around us is shaped in such a way that arms and hands are necessary to achieve most of daily-living activities; it is therefore unsurprising that the loss of the upper limb, partial or total, monoor bilateral, represents a severe impairment. Dexterous arm/hand prostheses for the upper-limb amputee as well as smart, natural control systems have been proposed, but reliability is still an issue: according to a comprehensive

All authors are with the Institute of Robotics and Mechatronics, DLR - German Aerospace Center, D-82234 Weßling, Germany.

* corresponding author: The author is also with the Neurorehabilitation Systems Research Group, Department of Trauma Surgery, Orthopedics and Plastic Surgery, University Medical Center Goettingen, 37075 Goettingen, Germany. email:gpatel@gwdg.de. literature review [1], self-powered prostheses rejection rates still are about one third for paediatric and one fourth for adult patients, and one of the major complaints about them regards the Human-Machine Interface (HMI) connecting the prosthetic artefact and the patient: it does not allow for simultaneous and proportional $(\mathrm{s} / \mathrm{p})$ control and lacks reliability [2], [3], [4], [5].

By reliability we mean that such an HMI should enable to grasp objects and operate tools exactly the way the subject wants to, and exactly for the time it is required. As opposed to that, state-of-the-art HMIs based upon surface electromyography (sEMG [6], [7]) still cannot avoid undesired openings/closings and/or wobbling while lifting a mug from a table to drink. Recommendations to alleviate this problem [8], [9], [10], [11] suggest that multi-modal sensing should be used, as well as incremental learning; the latter idea is particularly fascinating, since it entails that the subject could start interacting with the prosthesis rather than calibrating it once and for all in the beginning.

In this paper we take a slightly different approach to improving the reliability of hand prosthesis myocontrol. A specific learning method that we already used in the past can be exploited to enforce the composition of previous knowledge in order to reduce the number of patterns to be predicted, thus improving the reliability of the control system. Building upon our own previous work [12], we compare the standard usage of Ridge Regression with Random Fourier Features [13] (from now on called Full Regression, FR) with a modified version of the approach (from now on called Modular Regression, MR) in which specific models of a restricted set of actions can be built on-the-fly at low computational cost, though keeping the memory requirement bounded (i.e., independent of the number of input samples). For example, whereas a general model built using FR could contain patterns for several different ways of grasping, by exploiting MR we are able to instantaneously obtain a restricted model containing only the information related to, e.g., pointing the index and enforcing a lateral grip. This restricted MR-model has a clear application while withdrawing cash from an ATM, since only pointing the index (to enter one's own PIN on a keypad) and lateral 
grip (to insert/extract a credit card and to take the money) are required. In MR, previous knowledge does not need to be discarded: all patterns are stored in a database, and any subset of them can be cherry-picked on demand to best accomplish the task at hand.

We hypothesise that the systematic usage of MR would improve the accuracy of the prediction, therefore improving the reliability of the myocontrol system. In order to prove this claim, we engaged fourteen intact human subjects in a psychophysical experiment in which both FR and MR (in randomised order) were used while repeatedly performing actual daily-life tasks (e.g., ironing a cloth on an ironing board using a standard iron) with a commercially available multi-finger hand prosthesis mounted on a splint.

\section{A. Related Work}

S/p myocontrol, as opposed to sequential and discrete, was first defined in [14]; it is speculated in the community [10] that $\mathrm{s} / \mathrm{p}$ myocontrol enforces a better, more rewarding and natural experience for the user, and that it should be actively pursued [4]. One remarkable recent result can be found, for instance, in [15]. The machine learning method employed in our study has already been proved effective [12] in realising this kind of control, with the added value of being incremental, spacebounded, fast and non-linear. Incremental myocontrol is being exploited in assistive robotics by several groups in the world (e.g., [16]). Incremental learning is believed to lead to interaction with the control system [11], therefore improving the reliability of the prosthetic system.

The idea of exploiting knowledge composition in machine learning is not new, of course. Bottom line, single data sets for each action of interest can be gathered and then used to train a new model each time upon requirement; such approaches, however, are usually not incremental (i.e., their space occupancy grows as time passes and new samples must be recorded and stored in memory) and their training/prediction time grows with the required space; e.g., Support Vector Machines [17], [18] require $\mathcal{O}\left(n^{3}\right)$ for training and $\mathcal{O}(n)$ for prediction. The approach we propose in this work can be seen as a special case of knowledge transfer / adaptive learning, according to which information recorded during the synthesis of previous models should be re-used to boost the performance of the current model [19]. Our approach is, however, essentially different in that it presumes that model distilled using less information will perform better than larger ones, provided that no essential information is neglected.

\section{FULL REGRESSION AND MODULAR REGRESSION}

In $s / p$ myoelectric control [14] we try to model a function mapping bodily signals to graded prosthetic control commands for each degree of freedom (DOF) of a prosthesis. Typically, the inputs to such a function are vectors of features extracted from these signals, gathered via an array of sensors placed on a human subject's body; each vector is called a sample. The outputs are realvalued target values, representing the activations issued to the DOFs of the prosthesis; for example, current intensities to be applied to each motor. Mathematically speaking, $\mathbf{y}=f(\mathbf{x})$ where $\mathbf{x} \in \mathbb{R}^{d}$ and $\mathbf{y} \in \mathbb{R}^{M}$ represent the sample and target values respectively; and for our experiment, $d(=8)$ represents the number of sensors and $M(=6)$ represents the number of prosthesis motors. As is customary in supervised machine learning, in order to build $f$ we collect a training set of $N$ (sample,target) pairs $\mathcal{D}=\left\{\left(\mathbf{x}_{1}, \mathbf{y}_{1}\right), \ldots,\left(\mathbf{x}_{N}, \mathbf{y}_{N}\right)\right\} \triangleq$ $(X, Y)$, practically represented by a matrix $X \in \mathbb{R}^{N \times d}$ juxtaposing all samples and a further matrix $Y \in \mathbb{R}^{N \times M}$ juxtaposing the target values for each DOF, in the same order of the samples in $X$.

\section{A. Building the training set}

In practice, $\mathcal{D}$ must fairly represent $K$ signal patterns, corresponding to the different actions required by the subject, where typically, each action is a useful grasping (e.g., Power grasp, Pinch grip, etc.) or kinematic configuration (e.g., stretched hand, Pointing index, etc.). Target values appropriate for an action (ground truth) must be associated to each sample. Rather than employing further sensors to collect them, we hereby follow the so-called realistic or on-off goal-directed approach, already proved effective in, e.g., [20]: each novel pattern $j \in 1, \ldots, K$ is represented by a set of $N_{j}$ samples recorded from the subject while (s)he is stimulated to enforce the action, and each sample in the set is associated with a synthetic target value representing the minimal / maximal activation for each DOF of the prosthesis.

Let us sketch a running example. Consider the case of the $i$-LIMB Revolution five-fingered prosthetic hand manufactured by Touch Bionics (www.touchbionics.com), endowed with one motor for the flexion of each finger and one additional motor for the rotation of the thumb. In this case $M=6$ and we assume that the second component of the target value vector corresponds to thumb adduction and the third component corresponds to the motor controlling the index finger flexion. Assume, moreover, that we are interested in representing $K=3$ actions: the no-action (Rest) action, associated to minimal activation and thumb abduction, the Power 
grasp action, associated to maximal flexion of all fingers and thumb, and the Pointing index action, associated to maximal flexion of all fingers except for the index, which remains at minimal activation. In this case there are three target values, namely $\mathbf{y}_{r e}=\left[\begin{array}{llllll}0 & 0 & 0 & 0 & 0 & 0\end{array}\right]^{T}$, $\mathbf{y}_{p w}=\left[\begin{array}{llllll}1 & 0 & 1 & 1 & 1 & 1\end{array}\right]^{T}$ and $\mathbf{y}_{p o}=\left[\begin{array}{llllll}1 & 0 & 0 & 1 & 1 & 1\end{array}\right]^{T}$, and each sample is associated to one of them as follows:

$$
\mathcal{D}=\left(\left[\begin{array}{c}
X_{r e} \\
X_{p w} \\
X_{p o}
\end{array}\right],\left[\begin{array}{c}
Y_{r e} \\
Y_{p w} \\
Y_{p o}
\end{array}\right]\right)
$$

where $X_{j} \in \mathbb{R}^{N_{j} \times d}$ and $Y_{j} \in \mathbb{R}^{N_{j} \times 6} ; N=\sum_{j=1}^{K} N_{j}$ and each $Y_{j}$ consists of $N_{j}$ juxtaposed copies of the target value for the $j$ th action; for example,

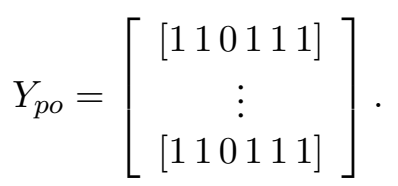

Notice that only minimal and maximal activations are considered, which greatly simplifies the subject's task; the regression interpolates the intermediate values. The on-off goal-directed approach simplifies the acquisition setup and the training phase for the subject, since (s)he is only supposed to enact $K$ actions for a short amount of time [20].

\section{B. Ridge Regression with Random Fourier Features}

In [12], [21] it has been demonstrated how the usage of a specific incremental machine learning method, namely Ridge Regression with Random Fourier Features (RR-RFF from now on), could be proficiently used in this setting to simultaneously and proportionally control a prosthetic hand in a teleoperated scenario. Ridge Regression (RR [22]), a regularised version of leastsquares regression, assumes a linear model $\mathbf{y}=W \mathbf{x}$, where $W \in \mathbb{R}^{M \times d}$. RR has a number of desirable characteristics that make it suitable for $\mathrm{s} / \mathrm{p}$ myocontrol: firstly, $W$ can be evaluated directly, avoiding the usage of optimisation methods:

$$
W=\left(X^{T} X+\lambda I_{d}\right)^{-1} X^{T} Y
$$

where $I_{d}$ is the identity martix of order $d$ and $\lambda>0$. The evaluation is relatively fast since it involves inverting a matrix of dimension $d$. Secondly, RR can easily be made incremental by means of a rank-1 update method such as, e.g., the Cholesky decomposition, so that it remains strictly bounded in space. Its memory occupancy is bounded by $d-$ it is independent from $N$, the number of samples the system has seen so far, entailing that it can in principle run forever. Thirdly, new target values $\mathbf{y}$ can be predicted by just evaluating $W \mathbf{x}$, which is a very fast operation. However, since the problem at hand is indeed non-linear [12], we rather seek a way to obtain a non-linear regressor while retaining all the desirable properties of RR. The most straightforward way is that of pre-processing each sample with a nonlinear mapping $\phi: \mathbb{R}^{d} \rightarrow \mathbb{R}^{D}$. The choice of $\phi$ is obviously crucial: in the case of RR-RFF [13], $\phi$ is the application of sinusoidal functions to the samples, weighted through randomly-sampled frequencies, inducing a finite-dimensional approximation of a Gaussian kernel. Let the matrix $\boldsymbol{\Phi} \in \mathbb{R}^{N \times D}$ be defined as

$$
\boldsymbol{\Phi} \triangleq\left[\begin{array}{c}
\phi\left(\mathbf{x}_{1}\right)^{T} \\
\vdots \\
\phi\left(\mathbf{x}_{N}\right)^{T}
\end{array}\right]
$$

then the model of RR-RFF evaluates to

$$
W=\left(\boldsymbol{\Phi}^{T} \boldsymbol{\Phi}+\lambda I_{D}\right)^{-1} \boldsymbol{\Phi}^{T} Y
$$

where, as opposed to Equation 3, $W \in \mathbb{R}^{M \times D}$. The target value for a new sample $\mathbf{x}$ is $\mathbf{y}=W \phi(\mathbf{x})$. Like RR, RR-RFF is fast both in the evaluation of the model and in prediction, can be made incremental and is bounded in space. RR-RFF can also be viewed as Linear Regression employing a specific finite $(D)$-dimensional kernel.

\section{Modular regression using $R R-R F F$}

The model obtained using Equation 4 employs $\boldsymbol{\Phi}$, which contains information regarding all $K$ actions such a model is the model of FR. However, as Equation 1 shows, $\mathcal{D}$ is naturally split across $K$ subsets $\left(\boldsymbol{\Phi}_{j}, Y_{j}\right)$ where $\boldsymbol{\Phi}_{j}=\phi\left(X_{j}\right)$, each one representing a single action. Equation 4 can be therefore rewritten as

$$
W=\left(\sum_{j=1}^{K} \boldsymbol{\Phi}_{j}^{T} \boldsymbol{\Phi}_{j}+\lambda I_{D}\right)^{-1} \sum_{j=1}^{K} \boldsymbol{\Phi}_{j}^{T} Y_{j}
$$

Since by Equation 2 each $Y_{j}$ is a juxtaposition of $N_{j}$ identical vectors composed of zeros and ones only, $\boldsymbol{\Phi}_{j}^{T} Y_{j}=\overline{\boldsymbol{\Phi}_{j}} \mathbf{y}_{j}^{T}$, where

$$
\overline{\mathbf{\Phi}_{j}} \triangleq \sum_{i=1}^{N_{j}} \phi\left(\mathbf{x}_{i j}\right)
$$

is the sum of all samples gathered during the enactment of the $j$ 'th action 17 Summing up, Equation 5 can be

\footnotetext{
${ }^{1}$ Notice that whenever $\mathbf{y}_{j}$ is identically null (in our example and typically, such a target value corresponds to the Rest state) the related

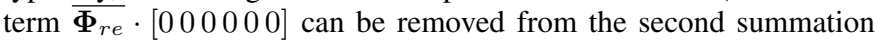
in Equation 5, leading to a further simplification.
} 
simplified to

$$
W=\left(\sum_{j=1}^{K} \boldsymbol{\Phi}_{j}^{T} \boldsymbol{\Phi}_{j}+\lambda I_{D}\right)^{-1} \sum_{j=1}^{K} \overline{\mathbf{\Phi}}_{j} \mathbf{y}_{j}^{T} .
$$

This way, in order to build $W$ we only need to store $K$ triplets $\left(\boldsymbol{\Phi}_{j}^{T} \boldsymbol{\Phi}_{j}, \overline{\mathbf{\Phi}_{j}}, \mathbf{y}_{j}\right), j=1, \ldots, K$, whose sizes do not depend on $N$. In the proposed approach, MR entails the possibility of building reduced models by plugging only some of the triplets into the previous equation. Whenever a particular task only requires a subset $K^{\prime}$ of the actions, such a reduced model $W_{K^{\prime}}$ can be evaluated on-the-fly: in fact, by Equation 6 it is straightforward that

$$
W_{K^{\prime}}=\left(\sum_{j \in K^{\prime}} \boldsymbol{\Phi}_{j}^{T} \boldsymbol{\Phi}_{j}+\lambda I_{D}\right)^{-1} \sum_{j \in K^{\prime}} \overline{\mathbf{\Phi}_{j}} \mathbf{y}_{j}^{T}
$$

for any $K^{\prime} \subseteq K$. Let us take back the example in Subsection II-A. The FR model, recognising all the three actions, is

$$
\begin{aligned}
& W_{r e, p w, p o}=\left(\boldsymbol{\Phi}_{r e}^{T} \boldsymbol{\Phi}_{r e}+\boldsymbol{\Phi}_{p w}^{T} \boldsymbol{\Phi}_{p w}+\boldsymbol{\Phi}_{p o}^{T} \boldsymbol{\Phi}_{p o}+\lambda I_{D}\right)^{-1}
\end{aligned}
$$

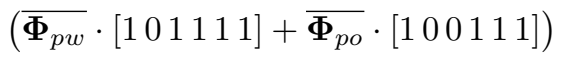

but we can also build, for instance, a MR model which will only recognise the Rest state and the Power grasp:

$$
\begin{aligned}
& W_{r e, p w}=\left(\boldsymbol{\Phi}_{r e}^{T} \boldsymbol{\Phi}_{r e}+\boldsymbol{\Phi}_{p w}^{T} \boldsymbol{\Phi}_{p w}+\lambda I_{D}\right)^{-1}
\end{aligned}
$$

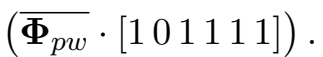

In practice, after having initially gathered and stored one triplet for each action, we can build one specific MR model $W_{K^{\prime}}$ for each situation the subject requires, instead of using the full model $W$. The computationally most costly operation is the inversion of a $D \times D$ matrix.

Lastly, notice that, due to the incrementality of the approach, it is possible (and desirable) to add further data to a single $\boldsymbol{\Phi}_{j}$ whenever it is required - for example, to counter the effects of muscle fatigue or because the subject has changed her/his signals in the course of time, due to the learning effect. From now on, each such reevaluation of the model will be called a model update.

\section{Materials AND Methods}

In order to check whether the usage of MR would yield a better myocontrol with respect to FR, we devised an experiment in which several intact human subjects would repetitively perform a set of grasping tasks using either method, in randomised order.

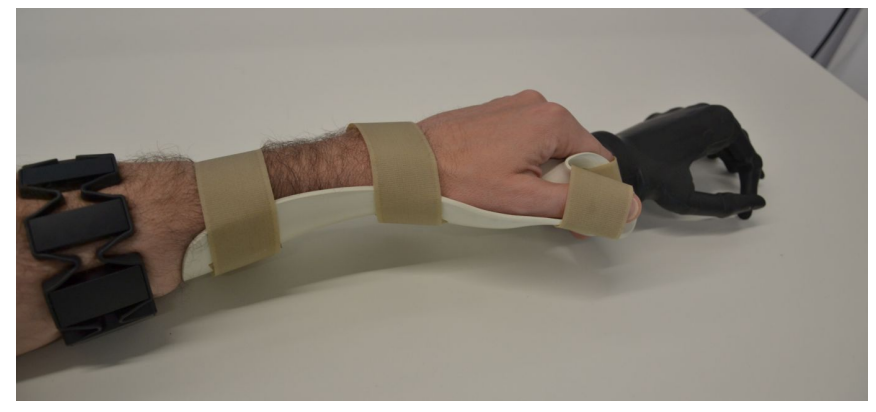

Fig. 1. The experimental setup, consisting of a Myo bracelet with eight sEMG sensors, a hand/wrist splint and an $i-L I M B$ prosthetic hand mounted on top.

\section{A. Participants}

Fourteen able-bodied subjects (age $24.79 \pm 2.52$ years, weight $76.71 \pm 7.3 \mathrm{~kg}$, all male, 13 right-handed and 1 left-handed) participated in the experiment, after being given an oral and written description of the experiment, and having signed an informed consent form. The experiment was conducted according to the WMA Declaration of Helsinki and previously approved by the DLR Ethical Committee.

\section{B. Experimental Setup}

The setup was designed to be light and wireless in order to provide the subjects with an as-natural-aspossible and exciting experience, bringing the experiment close to real life. sEMG signal gathering was enforced using a wireless commercial Myo bracelet manufactured by Thalmic Labs (www.myo.com) with eight channels, placed approximately $5 \mathrm{~cm}$ below the elbow joint of the left forearm. Additionally, each subject wore a hand/wrist splint, on top of which a left-sided $i$-LIMB Revolution hand prosthesis was fixed using a bolt and screw. A custom-built serial-over-Bluetooth circuit was used to control the hand wirelessly. The setup is depicted in Figure 1.

$\mathrm{S} / \mathrm{p}$ regression-based control was implemented as a stand-alone C\# program on a standard PC equipped with two Bluetooth dongles, the first to acquire raw sEMG data at $200 \mathrm{~Hz}$ from the Myo and the second to control the prosthetic hand. The software allowed gathering and storing the sEMG patterns and target values corresponding to each action, that is the triplets $\left(\boldsymbol{\Phi}_{j}^{T} \boldsymbol{\Phi}_{j}, \overline{\boldsymbol{\Phi}_{j}}, \mathbf{y}_{j}\right)$, furthermore it enforced the possibility of building a specific model for each task at hand, $W_{K^{\prime}}$. Each triplet was practically built using samples and target values collected for 5 seconds while contracting the forearm muscles to produce comfortably high fingertip forcepattern on the splint. The training data for each action was collected by verbally synchronizing the start of the 


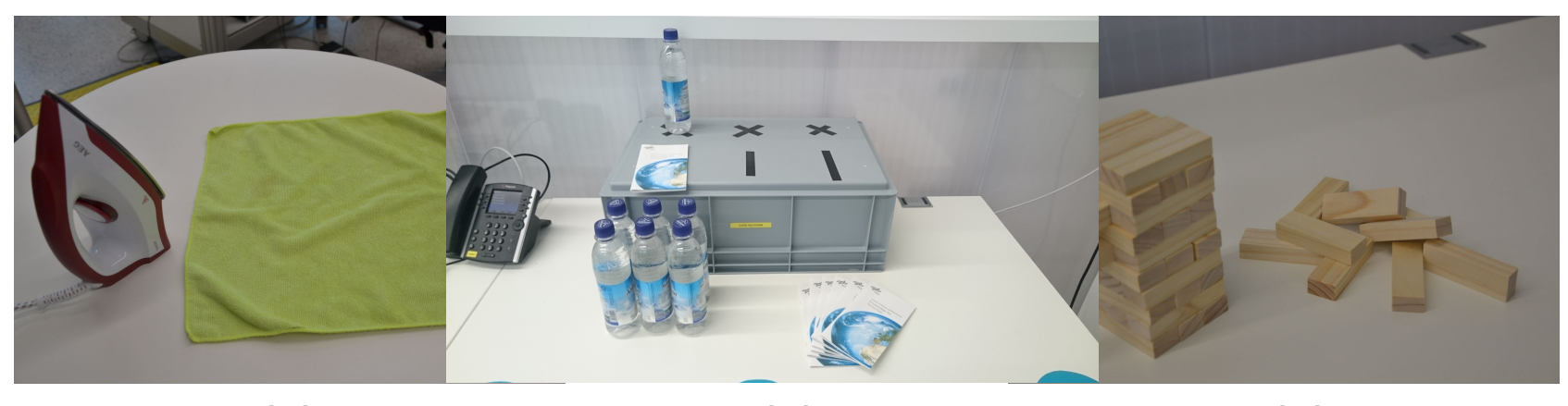

(A)

(B)

(C)

Fig. 2. The Figure shows the experimental setup for three different Tasks, namely Ironing (A), Arranging (B) and Jenga (C).

training phase, followed by the prosthesis emulating the action being trained. To indicate the end of the training phase, the prosthesis would release the configured action (being trained) and quickly go back to rest.

For each sEMG channel, the root mean square envelope was evaluated every $5 \mathrm{~ms}$ over a window size of $150 \mathrm{~ms}$ and then filtered using a low-pass Butterworth filter of order 1 with cutoff frequency set at $2 \mathrm{~Hz}$. The software suite enabled the experimenter to precisely time each task while keeping track of the errors and model updates required during its execution.

\section{Actions and Tasks}

The tasks required during the experiment were designed in order to reflect daily-living activities at home as well as outdoors; each task required, for its execution, a specific subset of $K=6$ actions mentioned below,

\begin{tabular}{|c|c|c|}
\hline Action & $\mathbf{y}_{\text {action }}$ & Description \\
\hline Power & {$\left[\begin{array}{llllll}1 & 0 & 1 & 1 & 1 & 1\end{array}\right]$} & $\begin{array}{l}\text { all fingers flexed, thumb abducted and } \\
\text { flexed }\end{array}$ \\
\hline Pinch & {$\left[\begin{array}{llllll}1 & 0 & 1 & 1 & 0 & 0\end{array}\right]$} & $\begin{array}{l}\text { Index and Middle finger flexed, thumb } \\
\text { abducted and flexed }\end{array}$ \\
\hline Point & {$\left[\begin{array}{llllll}1 & 0 & 0 & 1 & 1 & 1\end{array}\right]$} & $\begin{array}{l}\text { all fingers flexed but Index, thumb ab- } \\
\text { ducted and flexed }\end{array}$ \\
\hline Pre-Flat & {$\left[\begin{array}{lllllll}0 & 1 & 1 & 1 & 1 & 1\end{array}\right]$} & $\begin{array}{l}\text { all fingers flexed, thumb adducted but } \\
\text { not flexed }\end{array}$ \\
\hline Flat & {$\left[\begin{array}{lllllll}1 & 1 & 1 & 1 & 1 & 1\end{array}\right]$} & $\begin{array}{l}\text { all fingers flexed, thumb adducted and } \\
\text { flexed }\end{array}$ \\
\hline Rest & {$\left[\begin{array}{llllll}0 & 0 & 0 & 0 & 0 & 0\end{array}\right]$} & $\begin{array}{l}\text { all fingers at rest, thumb abducted but } \\
\text { not flexed }\end{array}$ \\
\hline
\end{tabular}

A description of each task of the experiment follows. Such tasks are inspired by those found in a number of existing functional assessment protocols such as ABILHAND[23], SHAP[24], UBET[25] and UNB[26], with a specific concern on bimanual ones requiring a minimum of two actions.

a) Ironing: A standard iron and a piece of square cloth were placed in front of the subjects as shown in Figure 2(A). The subjects were instructed to use a Power grasp to hold the iron and a Pinch grip for manipulating the cloth. First, the subjects had to iron the cloth four times right to left with the prosthetic hand; they were allowed to use their right hand to steadily hold the cloth while ironing. Next, the cloth was flipped using both hands and the back-side was again ironed four times. Finally, the cloth was folded twice (corner to corner) using both hands, reducing its size to one quarter of the original size. (For safety reasons, the iron was never turned on during the experiment.)

b) Library: A book was placed on the top compartment of a shelf and a starting point was marked approximately 4 meters away from the shelf; additionally, a desk with a keyboard was placed near the shelf, alongside the path between the shelf and the starting point. After walking towards the shelf from the starting point, the subjects grabbed the book using a Power grasp and placed it on the desk near the keyboard. Next, the subjects used a Point grip to enter the title of the book via the keyboard; lastly, before returning back to the starting point, the subjects placed the book back to its original location using a Power grasp.

c) Arranging: Figure 2(B) depicts the starting configuration of the task. Three pairs of crosses $(\times)$ and vertical lines $(\mid)$ were marked on an alleviated platform. The task consisted of placing two flyers and two bottles each on the $\mid$ and $\times$ markers respectively. One set of a bottle and a flyer was already on the first marker pair to act as a visual clue. In the beginning of the task, subjects took six flyers in their right hand and thereafter, they used a combination of Pre-Flat and Flat grasp to pickand-place two flyers from the right hand onto the vertical line (|) markers. To accomplish the flyer pick-and-place task, first the Pre-Flat grip was executed and then a flyer was placed between the thumb and the radial side of the index finger, before closing the thumb to execute the Flat grasp. Additionally, six bottles in a block of $3 \times 2$ were placed on the table between the alleviated platform and 


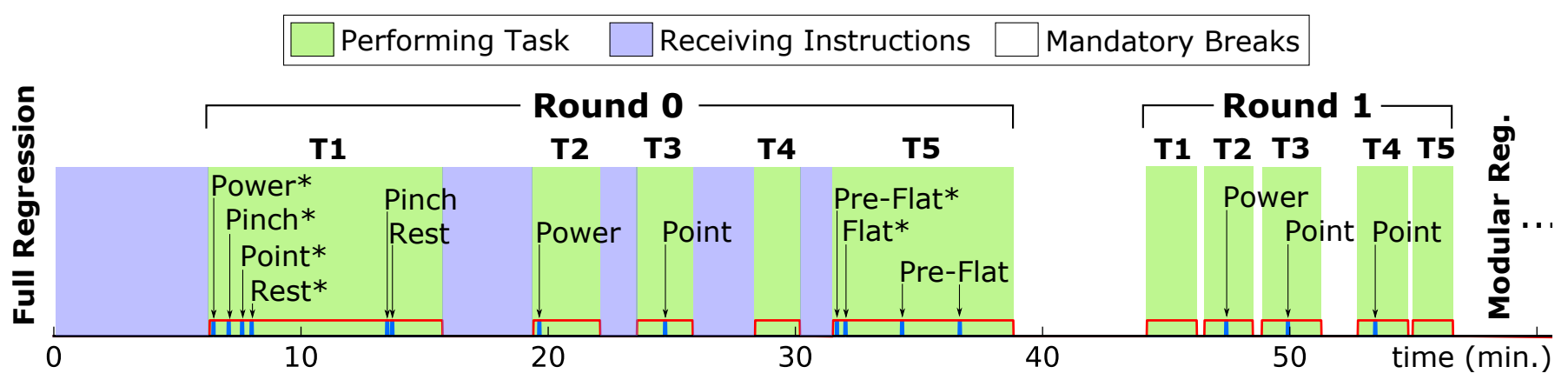

Fig. 3. A typical experimental timeline. The subject starts in the FR modality and performs five tasks (T1-Shopping, T2-Ironing, T3-Library, T4-Jenga and T5-Arranging); occasionally, model updates are requested (a star denotes mandatory updates, that is, updates for actions that had never been trained in the past and the event of model update has been indicated using a blue marker along the timeline).

the subject, who then used the Power grasp to pick-andplace any two bottles on the cross $(x)$ markers (from the table onto the alleviated platform). In case, if the subjects would lose the grasped bottle during pick-andplace (e.g. due to accidental opening), they would grasp a new bottle from the block of bottles placed in front of them.

d) Shopping: Three items, namely, water-bottles, fruits (bananas) and chocolates were placed on a shelf at different heights and a starting point was marked approximately 4 meters away from the shelf; here, a standard shopping basket was given to the subjects in their right hand. A keyboard (on a desk) was placed alongside the shelf and the starting point. At first, the subjects walked towards the shelf with the basket in their right hand and then, used their prosthetic hand to pick-and-place items from the shelf into the basket. The subjects used a Power grasp to pick-up a single bottle, a Pinch grip to pick-up a single fruit and a Point grip to slide and take a single chocolate. Next, they used a Point grip to enter a PIN (1973) on the keyboard, before walking back towards the starting point.

e) Playing Jenga: As shown in Figure 2(C), a small tower with 30 blocks was pre-constructed for the subjects, with two blocks coming out of the tower on the left-side. Firstly, using a Point grip, the subjects were asked to push the two blocks inwards, until a part of the block comes out on the right-side. Next, with the sound hand, the subjects removed the two blocks from the right-side of the tower. Finally, they used a Pinch grip to pick-and-place two blocks on top of the tower from the pile of blocks on the right.

\section{Timeline}

Firstly, each subject was briefly introduced to the concept of myoelectric control using regression and informed that the experiment was tailored to compare two different regression approaches; however, the aim of the experiment (e.g., to test whether one approach was better than the other one, and in which sense) was not disclosed, in order not to generate any expectation bias. Soon after, the experiment would begin: for each of the two modalities (FR and MR), the subjects were challenged to perform two rounds (Round 0 and Round 1) each comprising a single repetition of the five tasks. The sequence in which the two modalities were administered was randomized across subjects, i.e., half of them used FR followed by MR, while the other half had the order switched; as well, the order of the tasks was randomised across subjects.

During Round 0, the subjects received extensive advice and instructions on how each task was to be completed, in order to familiarise them with the tasks and setup. While executing each task for the first time, the six triplets would be gathered in an incremental fashion, i.e., training only-upon-requirement until all five tasks were successfully completed in Round 0 . During Round 1 the tasks were again administered, while three measures of performance were noted down: the Time to Complete a Task (TCT) in Round 1, the number of Action Updates (AUs i.e. model update for a given action) requested by the subject in Round 1 and the number of errors incurred during the task (Err.). The measure Err. was the count of accidental dropping of grasped object during each task and the accidental opening of the hand while typing in the Library and Supermarket task. The model evaluated during Round 0 was reused in Round 1 and the subjects were always allowed to incrementally add more training data to the model. In Round 0, the decision to perform an AU was collectively taken by the subject and the experimenter via verbal interaction, whereas in Round 1, the request to perform $\mathrm{AU}$ was independently expressed by the subject.

To better understand the approach, consider Figure 3. 


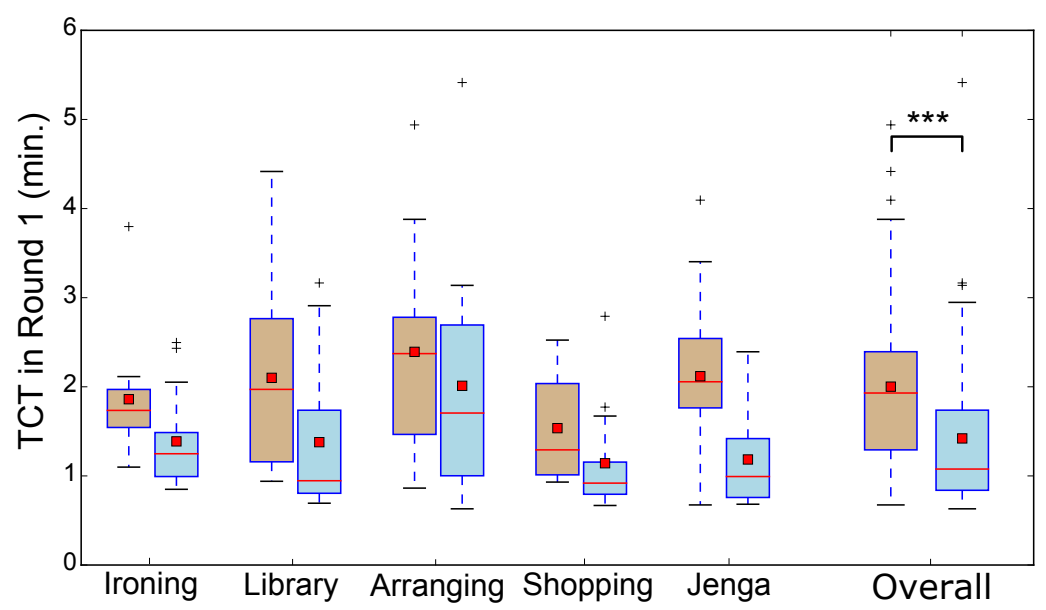

(A)

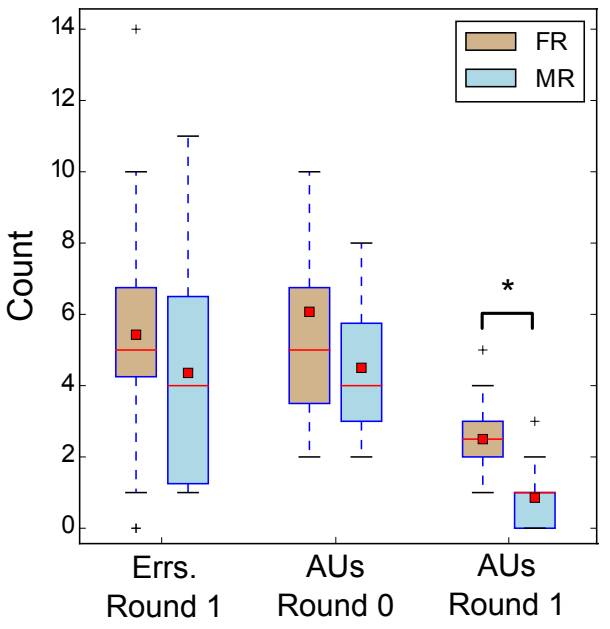

(B)

Fig. 4. Summary of the comparison between FR and MR. (A) median Time to Complete a Task (TCT) in Round 1 in minutes. (B) median count of the errors (Err.) during Round 1 and median count of action updates (AUs) in Round 0 and Round 1; one outlier with 18 AUs in Round 0 with FR has not been shown. (***, $p<0.001$; **, $p<0.01{ }^{*}, p<0.05$; '+', represents outliers; 'red square', population mean; 'red line', population median)

representing a typical experimental timeline. The subject first performs Task T1 and needs to train once the actions required for it (Power, Pinch, Point and Rest). Later on, due to poor performance, two model updates for the action Pinch and Rest were performed. After T1 ends successfully, T2, T3 and T4 are smoothly performed, although an update for the action Power (during T2) and for Point (during T3) were performed. For T5, the actions Pre-Flat and Flat must be initially acquired, as they were not required for T1-T4, then during the execution of the task two more updates for action Pre-Flat were executed. Round 1 follows (notice that it takes remarkably less time, due to the familiarisation occurred during Round 0 ), during which only three more updates were requested, namely for Power (T2), Point (T3) and Point again (T4).

Whenever the FR modality was being tried, a single model evaluated using all triplets available was used to control the prosthetic hand; that means that the subjects always had all actions at their disposal. As opposed to this, in the MR modality the experimenter would select the appropriate subset $K^{\prime}$ of triplets before each new task started, restricting the number of actions the subjects were able to perform to those strictly necessary for the task.

\section{E. Data Analysis}

The data of different outcome measures (e.g. TCT, AUs, Err.) did not pass the test for normality and hence, nonparametric statistics was used to compare the two regression approaches FR and MR. The time duration required to accomplish a given task in Round 1 was compared using the Sign test for dependent samples with Bonferroni correction of alpha. The outcome measures AUs in Round 0, AUs in Round 1 and Err. in Round 1 were individually compared between the FR and MR modality using the Sign test for dependent samples. Additionally, the measure AUs in Round $O$ was again compared using the Sign test for dependent samples with Bonferroni correction of alpha. All results are reported in terms of median and interquartile range (IQR). The software STATISTICA (Dell, US) was used to preform the statistical analysis and the threshold for significance was set at $\alpha=0.05$.

\section{EXPERIMENTAL RESUlts}

Time to Complete a Task: Figure 4(A) compares the median TCT in Round 1 using either FR or MR. For the factor Regression Type, the median overall duration required to complete the tasks using MR was significantly lower than using FR $(1.1[I Q R: 0.8-1.7]$ minutes versus $1.9[I Q R: 1.3-2.4]$ minutes, $p<0.001)$. Next, the average overall TCT with FR and MR was $2.0 \pm 0.9$ and $1.4 \pm 0.8$ minutes respectively, and this corresponds to an average overall percentage decrease of $30 \%$ with MR.

Errors: Figure 4(B) (leftmost bar) summarises the median number of errors during Round 1 for FR and MR. No statistically significant difference was observed between the two cases. The medain number of errors in Round 1 using the FR and MR were 5 [IQR:4.3-6.8] 
and $4[I Q R: 1.3-6.5]$, respectively. If the error count is normalized by the number of task (i.e. 5), it approximates to about one error per task. Thus, the error count per task is too small to make any meaningful comparison regrading the robustness of either approach. During the experiments, it was observed that the subjects were cautious and reluctant to pick up an object if they felt that they would lose it while manipulation.

Action Updates: As shown in Fig. 4(B), the number of updates/retraining required in Round 0 for the FR ( 5 $[I Q R: 4.3-6.8])$ and the MR (4 [IQR : $3.0-5.7])$ were not significantly different, whereas the amount of retraining required in Round 1 with the MR (1 $[I Q R: 0-1])$ was significantly lower than with the FR $(2.5[I Q R: 2-3])$. The number of action updates in Round 0 was depended on individual patterns. As seen in Fig 5, the number of times the pattern Rest was retrained was significantly higher than any other pattern except Point and Power. Next, the action Point was retrained more as compared to the patterns Pinch and Flat; and the actions Pinch and Power were retrained more in comparison to Flat. Thus, the number of AUs in Round 0 were independent of the type of Regression used (Fig 4(B)), but were rather depended on individual actions (Fig 5). This observation is likely because, the model for prediction was trained from scratch during Round 0 and the training data of each action in all required hand configurations/positions was not available. Whereas in Round 1, the regression model contained the training data of each action in all required hand configurations and hence, lower updates were required in Round 1 with MR versus FR (Fig 4(B)).

As discussed previously, the subjects were anonymously administered the two Regressors in a random order. At the end of the experiment, they were asked about their impression/feeling on which approach they thought was a better myoelectric control. 12 out of 14 subjects reported that the myoelectric control in the rounds with MR was better and 2 subjects reported perceiving no difference between the two approaches. These 2 subjects belonged to the group that was administrated MR followed by FR.

\section{Discussion}

Summing up the results of the statistical analysis we can say that the Modular Regression significantly improves the performance for every day tasks. Overall, the five tasks chosen in this experiment could be performed significantly faster using MR than FR. Furthermore, the MR control proved to be more reliable, since it required significantly less updates during the task execution in Round 1.

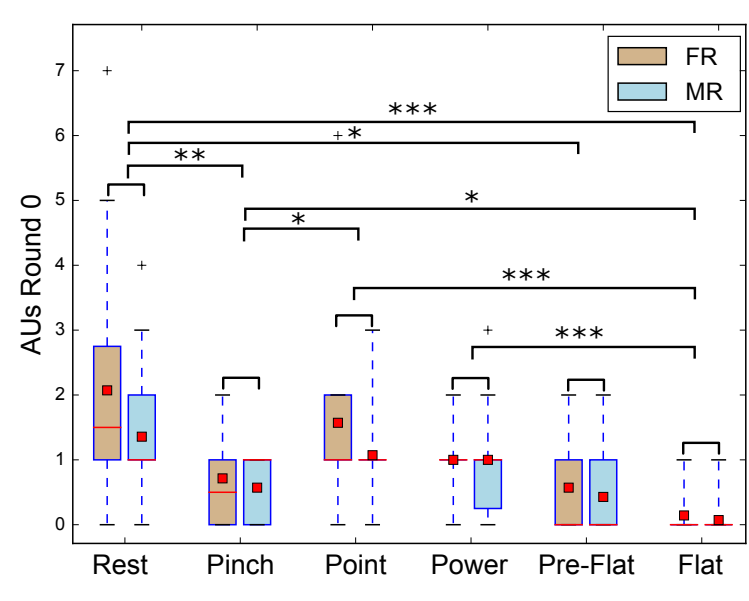

Fig. 5. The Action Updates in Round 0 with FR and MR for six different actions Rest, Point, Pinch, Power, Pre-Flat and Flat. $(* * *, p<0.001$; **, $p<0.01$; *, $p<0.05$; '+', represents outliers; 'red square', population mean; 'red line', population median)

The method presented in this study offers the potential to greatly simplify the interaction with the myoelectric control. At any given time the users can decide whether they require full control of the prosthesis or only require a reduced amount of actions. Such a reduced action set allows for a more precise and more stable control, since interaction with undesired action are eliminated. As depicted in Figure 6, the pattern overlap between different actions is much higher with FR in comparison to MR. The lower the overlap the better the ML algorithm can separate or detect the correct actions. Taking for example Figures 6(A) and 6(B) we can see that trying to perform the Pinch action in the situation depicted in Figure 6(A) would probably result in problems due to an unwanted interaction with the Point action. For the situation in Figure 6(B), this would not be the case. We assume we want to perform the ironing task and therefore we do not want to predict the Point action at all. This potentially gives the users more confidence in using their myoelectric prosthetic hand.

Not only can the subsets be chosen willingly, but the prosthetic device can potentially recognise situations and automatically apply a reduced control approach tailored to said/detected situation.

\section{A. Autonomous Context-Aware Adaptation}

Let us return to the example from the beginning, withdrawing money. One can imagine that approaching an ATM could be a situation that can be automatically detected using e.g. vision based methods. By doing so the intelligent control method could assume that 


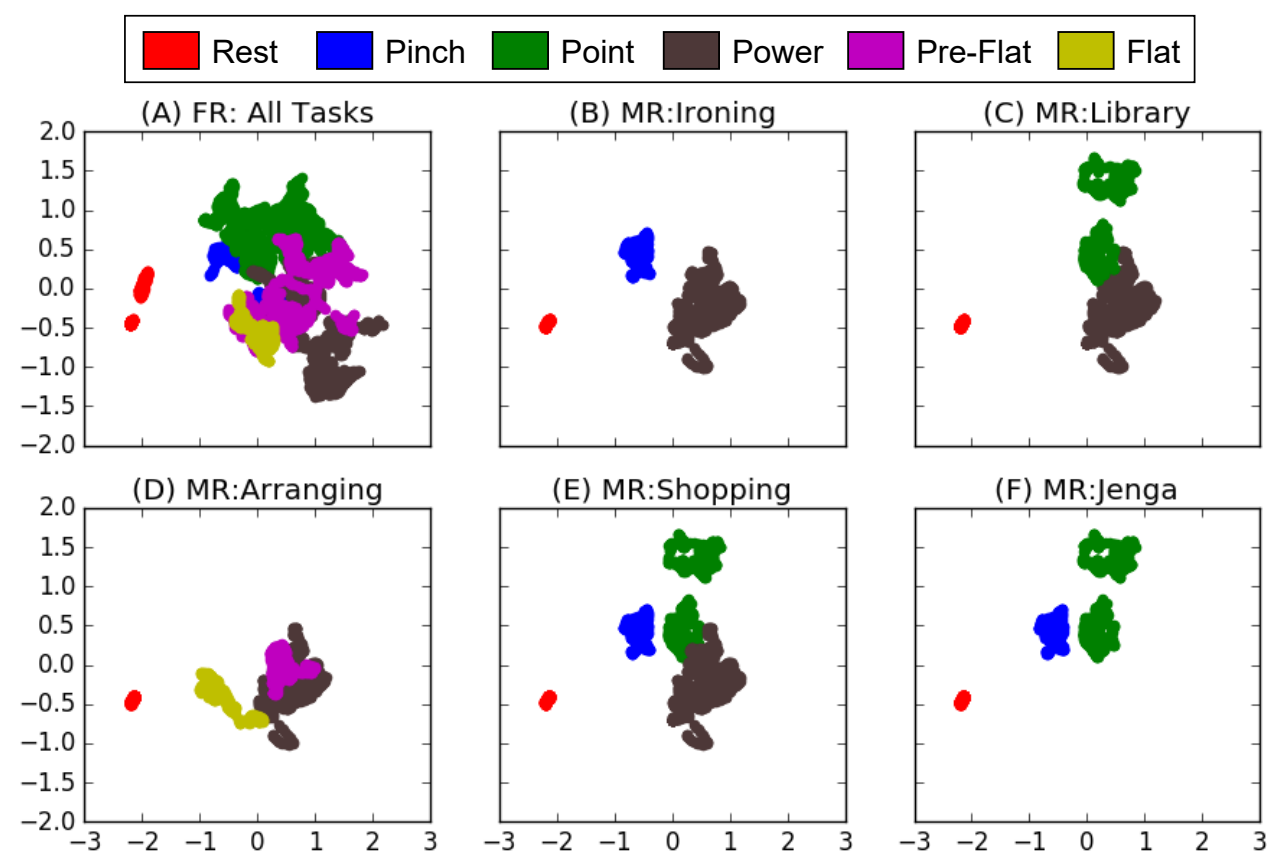

Fig. 6. Visualisation of training data used to generate the regression model when performing different tasks with FR (A) and MR (B-F). Principle Component Analysis (PCA) was used to reduce the dimensions of training data and the explained variance for the plotted data is $72 \%$. (The plotted training data was collected on a single subject, whose experimental timeline is shown in Figure 3 )

Pointing and Flat grasp are more likely to occur and can autonomously generate a model to predict on the reduced set of required actions.

A further application could include IMU data, which could be used to compensate for the changes in the sEMG signal depending on the position and orientation of the arm. Assuming training data $\left(\boldsymbol{\Phi}_{j}^{T} \boldsymbol{\Phi}_{j}, \overline{\boldsymbol{\Phi}_{j}}\right)$ for different arm position is available, using equation 6, a new model for prediction could be automatically generated, when a sufficiently large change in position/orientation is detected. A non-automated implementation of this idea showed promising results for a LDA based myoelectric control [27].

\section{B. User Adaptability}

Considering Eq. 6 there is a further possibility of modularly adapting the regression algorithm. The target values $\mathbf{y}_{j}$ can be modified independently from the training data $\left(\boldsymbol{\Phi}_{j}^{T} \boldsymbol{\Phi}_{j}, \overline{\boldsymbol{\Phi}_{j}}\right)$. Let us assume a user that is not able to consistently express more than 3 different sEMG pattern. In this study alone we used 5 different grasp patterns. Said user would not be able to perform all the tasks in this study using FR. On the other hand, since neither of the tasks requires more than 3 grasps, we could simply use the 3 different sEMG pattern that the user can express and map them to the current subset of tasks. This can easily be done by adapting the target values $\mathbf{y}_{j}$ of Eq. 6 .
This, unfortunately, goes against our goal of an intuitive control modality, since depending on the situation a certain muscle activation would result in different hand motions. On the other hand, this modification allows users to perform a higher number of hand activation than they would be able to control with a traditional algorithm (here e.g. FR). Further investigations are required to clearly determine the benefits and drawbacks of such a non-intuitive approach.

\section{User Interaction}

The clinical state of the art for myoelectric control is still a 2 sEMG electrode setup, where one electrodes is used for opening the prosthetic hand and one for closing it [28]. In order to realise more than just one hand gesture (open/close) a mode switching motion has to be performed. In case of a multi-articulated hand such as the i-LIMB Revolution by TouchBionics, a variety of motions can be performed. Cycling through all of these motions is cumbersome, therefore Touch Bioncs provides a smartphone app to select grasps with one's sound hand [29].

Although this requires a second hand, the interactive aspect and the possibilities of customisation are very welcome among users. The approach presented in this study allows for the same level of interaction and customisation and even more. While the TouchBionics app allows only to open and close one grasp at a time, 
our methods allows the users to customize several hand gestures for simultaneous and proportional control.

\section{Conclusion}

Modular Regression enables the user to perform several actions tailored to the situation at hand. This method proved superior to the traditional regression method, here FR. More and more confidence is placed in ML-based myoelectric control for prosthesis, which can be seen by the advent of such control algorithms in the commercial market [29], such as the COAPT system [30]. The modular approach of pattern recognition presented here can be used to enhance available prosthesis control schemes and, thus, will allow the users to have a more precise control of their hands and shall make ML-based myoelectric control more interactive, thereby, improving the overall end-user experience.

\section{ACKNOWLEDGMENTS}

The authors want to thank Mr. Bernd Henze, Mr. Alexander Werner and Dr. Maximo Roa of the DLR for providing support in the construction of the setup. The graphs and figures presented in the manuscript were created using Inkscape (www.inkscape.org) and Matplotlib with Python 3.3 (www.matplotlib.org, www.python.org).

\section{REFERENCES}

[1] E. Biddiss and T. Chau, "Upper-limb prosthetics: critical factors in device abandonment," Am J Phys Med Rehabil, vol. 86, no. 12, pp. 977-987, 2007.

[2] S. Micera, J. Carpaneto, and S. Raspopovic, "Control of hand prostheses using peripheral information," IEEE Reviews in Biomedical Engineering, vol. 3, pp. 48-68, Oct. 2010.

[3] P. J. Kyberd and W. Hill, "Survey of upper limb prosthesis users in sweden, the united kingdom and canada," Prosthetics and Orthotics International, vol. 35, no. 2, pp. 234-241, 2011.

[4] B. Peerdeman, D. Boere, H. Witteveen, R. H. in 't Veld, H. Hermens, S. Stramigioli, H. Rietman, P. Veltink, and S. Misra, "Myoelectric forearm prostheses: State of the art from a usercentered perspective," Journal of Rehabilitation Research and Development, vol. 48, no. 6, pp. 719-738, 2011.

[5] S. M. Engdahl, B. P. Christie, B. Kelly, A. Davis, C. A. Chestek, and D. H. Gates, "Surveying the interest of individuals with upper limb loss in novel prosthetic control techniques," Journal of NeuroEngineering and Rehabilitation, vol. 12, no. 1, pp. 111, 2015.

[6] R. Merletti, A. Botter, C. Cescon, M. Minetto, and T. Vieira, "Advances in surface EMG: Recent progress in clinical research applications," Critical reviews in biomedical engineering, vol. 38, no. 4, pp. 347-379, 2011.

[7] R. Merletti, M. Aventaggiato, A. Botter, A. Holobar, H. Marateb, and T. Vieira, "Advances in surface EMG: Recent progress in detection and processing techniques," Critical reviews in biomedical engineering, vol. 38, no. 4, pp. 305-345, 2011.
[8] N. Jiang, S. Dosen, K.-R. Müller, and D. Farina, "Myoelectric control of artificial limbs: is there the need for a change of focus?" IEEE Signal Processing Magazine, vol. 29, no. 5, pp. 149-152, 2012.

[9] M. Ison and P. Artemiadis, "The role of muscle synergies in myoelectric control: trends and challenges for simultaneous multifunction control," J. Neural Eng., vol. 11, 2014.

[10] A. Fougner, Ø. Stavdahl, P. J. Kyberd, Y. G. Losier, and P. A. Parker, "Control of upper limb prostheses: Terminology and proportional myoelectric control - a review," IEEE Trans Neur Syst Rehab Eng, vol. 20, no. 5, pp. 663-677, 2012.

[11] C. Castellini, R. M. Bongers, M. Nowak, and C. K. van der Sluis, "Upper-limb prosthetic myocontrol: two recommendations," Frontiers in Neuroscience, vol. 9, no. 496, 2015.

[12] A. Gijsberts, R. Bohra, D. Sierra González, A. Werner, M. Nowak, B. Caputo, M. Roa, and C. Castellini, "Stable myoelectric control of a hand prosthesis using non-linear incremental learning," Frontiers in Neurorobotics, vol. 8, no. 8, 2014.

[13] A. Rahimi and B. Recht, "Uniform approximation of functions with random bases," in Allerton Conference on Communication Control and Computing (Allerton08), Sep. 2008, pp. 555-561.

[14] N. Jiang, P. Parker, and K. Englehart, "Extracting simultaneous and proportional neural control information for multiple degree of freedom prostheses from the surface electromyographic signal," IEEE Transactions on Biomedical Engineering, vol. 56, no. 4, pp. 1070-1080, 2009.

[15] S. Amsuess, P. Goebel, B. Graimann, and D. Farina, "A multiclass proportional myocontrol algorithm for upper limb prosthesis control: Validation in real-life scenarios on amputees," Neural Systems and Rehabilitation Engineering, IEEE Transactions on, vol. 23, no. 5, pp. 827-836, Sept 2015.

[16] M. Rasouli, R. Ghosh, W. W. Lee, N. Thakor, and S. Kukreja, "Stable force-myographic control of a prosthetic hand using incremental learning," in Engineering in Medicine and Biology Society $(E M B C), 2015$ 37th Annual International Conference of the IEEE, Aug 2015, pp. 4828-4831.

[17] B. E. Boser, I. M. Guyon, and V. N. Vapnik, "A training algorithm for optimal margin classifiers," in Proceedings of the 5th Annual ACM Workshop on Computational Learning Theory (COLT), D. Haussler, Ed. ACM press, 1992, pp. 144-152.

[18] C. J. C. Burges, "A tutorial on Support Vector Machines for pattern recognition," Knowledge Discovery and Data Mining, vol. 2, no. 2, 1998.

[19] T. Tommasi, F. Orabona, C. Castellini, and B. Caputo, "Improving control of dexterous hand prostheses using adaptive learning," IEEE Transactions on Robotics, vol. 29, no. 1, pp. 207-219, 2012.

[20] D. Sierra González and C. Castellini, "A realistic implementation of ultrasound imaging as a human-machine interface for upper-limb amputees," Frontiers in Neurorobotics, vol. 7, no. 17, 2013.

[21] I. Strazzulla, M. Nowak, M. Controzzi, C. Cipriani, and C. Castellini, "Online bimanual manipulation using surface electromyography and incremental learning," IEEE Transactions on Neural Systems and Rehabilitation Engineering, 2016, in press.

[22] A. E. Hoerl and R. W. Kennard, "Ridge regression: Biased estimation for nonorthogonal problems," Technometrics, vol. 12, pp. 55-67, 1970.

[23] M. Penta, J.-L. Thonnard, and L. Tesio, "Abilhand: A rasch-built measure of manual ability," Archives of Physical Medicine and Rehabilitation, vol. 79, no. 9, pp. 1038 1042, 1998. [Online]. Available: http://www.sciencedirect.com/ science/article/pii/S0003999398901678 
[24] C. M. Light, P. H. Chappell, and P. J. Kyberd, "Establishing a standardized clinical assessment tool of pathologic and prosthetic hand function: Normative data, reliability, and validity," Archives of Physical Medicine and Rehabilitation, vol. 83, no. 6, pp. 776 - 783, 2002. [Online]. Available: http:// www.sciencedirect.com/science/article/pii/S0003999302095795

[25] A. M. Bagley, F. Molitor, L. V. Wagner, W. Tomhave, and M. A. James, "The unilateral below elbow test: a function test for children with unilateral congenital below elbow deficiency," Developmental Medicine \& Child Neurology, vol. 48, no. 7, pp. 569-575, 0072006.

[26] E. Sanderson and R. Scott, "Unb test of prosthetics function: a test for unilateral upper extremity amputees, ages 2-13," Fredericton (Canada): Institute of Biomedical Engineering, University of New Brunswick, 1985.

[27] A. Fougner, E. Scheme, A. D. C. Chan, K. Englehart, and . Stavdahl, "Resolving the limb position effect in myoelectric pattern recognition," IEEE Transactions on Neural Systems and Rehabilitation Engineering, vol. 19, no. 6, pp. 644-651, Dec 2011.

[28] P. Parker, K. Englehart, and B. Hudgins, "Myoelectric signal processing for control of powered limb prostheses," Journal of electromyography and kinesiology, vol. 16, no. 6, pp. 541-548, 2006.

[29] I. Vujaklija, D. Farina, and O. Aszmann, "New developments in prosthetic arm systems," Orthopedic Research and Reviews (in Press), 2016.

[30] C. LLC, “COAPT Complete Control," May 2016. [Online]. Available: https://www.coaptengineering.com/

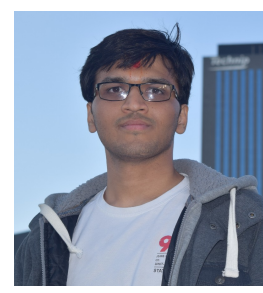

Gauravkumar K. Patel (MSc.) received his MSc. in Communication Engineering from RWTH Aachen University, Germany. During his academics, he focused mainly on concepts of machine learning and signal processing. He did an internship on myoelectric control for upper-arm prosthesis with the Robotics and Mechatronics Center of the German Aerospace Center (DLR) in Oberpfaffenhofen. Currenlty, he is pursuing a $\mathrm{PhD}$ at the Georg-August-University in Goettingen and he is also collaborating with DLR.

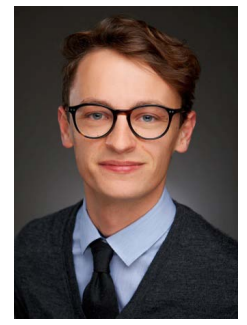

Markus Nowak (Dipl.-Ing.) received his M.Sc. degree in mechanical engineering from the Technical University of Munich (TUM). In his masters curriculum he focused on medical engineering and numerical mechanics. His master thesis was carried in cooperation with the Robotics and Mechatronics Center of the German Aerospace Center (DLR) in Oberpfaffenhofen. After his masters he continued as a research fellow at the Robotics and Mechatronics Center with focus on human-machine interfaces for controlling upper-limb prosthetics.

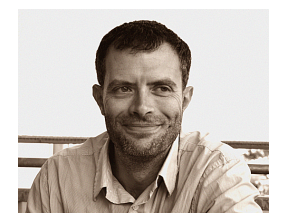

Claudio Castellini, Ph.D. received a Laurea in Biomedical Engineerings in 1998 from the University of Genova, Italy and a Ph.D. in Artificial Intelligence in 2005 from the University of Edinburgh, Scotland. Since 2009 he is a researcher at the DLR (German Aerospace Center) in Oberpfaffenhofen, concentrating on human-machine interfaces for the disabled and assistive robotics. He is currently (co)author of some 75 papers appeared in international journals, books and peerreviewed conferences. 